\title{
A conference update
}

\author{
Technology breakthroughs at the 2021 Symposia on VLSI Technology and Circuits.
}

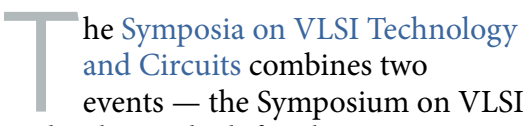

Technology, which first began in 1981, and the Symposium on VLSI Circuits, which began in 1987 - and provides a venue for all those in the business of VLSI (or very large-scale integration). Building on our annual coverage of the IEEE International Electron Devices Meeting (IEDM), last year we highlighted some of the breakthroughs reported at the 2020 VLSI Symposia. This year we return to the event, which took place online last month, to offer our highlights of the 2021 symposia.

We start with work on the future of advanced complementary metal-oxidesemiconductor (CMOS) logic. Such technology currently relies on fin-shaped transistors, but these devices may shortly be replaced with gate-all-around nanosheet transistors that typically consist of three or four stacked nanosheets. Chee-Wee Liu and colleagues at the National Taiwan University have now developed germanium-based eight-stack nanosheet transistors, as well as seven-stack nanowire gate-all-around devices.

Next we move to work on the future of memory. Magnetoresistive random-access memory (MRAM) stores information in elements known as magnetic tunnel junctions, which can be electrically switched via an effect known as spin-transfer torque (STT). STT-MRAM is a potential replacement for conventional technologies such as static random-access memory (SRAM), offering improved performance and smaller cell sizes. Mikio Oka and colleagues at Sony have now created a $30 \mathrm{Mbit}$ embedded STT-MRAM that can be used for buffer memory in three-dimensionally stacked CMOS image sensors.

In-memory computing is an approach that uses memory devices for both data storage and processing. It is of particular interest in the development of hardware for deep neural networks, which rely on multiply-accumulate operations. Pritish Narayanan and colleagues at IBM Research have now produced an in-memory computing chip that is based on analogue phase-change memory devices fabricated in $14 \mathrm{~nm}$ CMOS technology and can perform multiply-accumulate operations with high accuracy.

We then highlight work from Jahnavi Sharma and colleagues on the potential of integrated optical input-output technologies in high-speed communications. The team, who are based at Intel, have developed a hybrid integrated transceiver in which an electronic integrated circuit consisting of four microring modulator drivers - and fabricated with $28 \mathrm{~nm}$ CMOS technology - is combined with a photonic integrated circuit containing an array of microring modulators.

We also highlight work from Fukashi Morishita and colleagues on distributed camera systems that can operate round the clock - and thus need to have high sensitivity in low-light conditions. The researchers, who are based at Renesas Electronics Corporation in Japan, have created a high-sensitivity CMOS image sensor, which can be combined with an artificial intelligence accelerator to perform deep-neural-network-based image processing at the edge (that is, at the point of data collection). Finally, we highlight work from Nachiket Desai and colleagues at Intel on high-frequency power converter modules made from gallium nitride high-electron-mobility transistors.

Owing to the ongoing coronavirus pandemic, the 2021 Symposia on VLSI Technology and Circuits was held entirely as a virtual event. For researchers in certain countries, life is returning to a semblance of normality and conference organizers are cautiously exploring a return to in-person events. We (the editors of Nature Electronics), together with editors from Nature Nanotechnology and Nature Sustainability, as well as Korea University and APRU Sustainable Waste Management, are, for instance, currently organizing a conference that is planned as a hybrid event: in other words, researchers can attend in-person or online. The conference will take place later this year on the 26-28 October and will be located in Seoul, South Korea. Entitled Waste Management and Valorisation for a Sustainable Future, the event will explore sustainable waste management strategies and, among other challenges, consider the problem of - and potential solutions to - electronic waste. And looking further ahead, IEDM 2021, which takes place in December, is currently planned as an in-person event in its usual San Francisco location.

Published online: 26 July 2021

https://doi.org/10.1038/s41928-021-00623-8 